\title{
Hemodynamics of human placenta
}

\author{
E. S. Bernad ${ }^{1}$, M. Craina ${ }^{1}$ \& S. I. Bernad ${ }^{2}$ \\ ${ }^{1}$ University of Medicine and Pharmacy Timisoara, Romania \\ ${ }^{2}$ Romanian Academy, Timisoara Branch, Romania
}

\begin{abstract}
The placenta is responsible for the exchange of oxygen and nutrients from the mother to the fetus. Normal placentation and placental development are critical for a successful pregnancy. In this study the placenta are obtained from normal pregnancy and of normal vaginal delivery (age: 31 years old, parity 1, and gestation 38 weeks). After the delivery of the baby and the placenta commercial polymer mixture was injected into the artery to achieve a placental vascular model. The polymeric cast of the fetal vasculature of full-term placenta is used in order to perform a numerical simulation of the blood perfusion in the placenta. The period for the simulations is $0.5 \mathrm{~s}$, which corresponds to a baby heart rate of $120 \mathrm{bpm}$. Numerical simulation indicated that the velocity profiles in the placental vessels, both immediately upstream and downstream the bifurcation, are close to parabolic profiles.

Keywords: placenta, numerical simulation, fetoplacental vasculature.
\end{abstract}

\section{Introduction}

The placenta is an essential organ for normal development of the fetus, and accordingly, inadequate blood perfusion may lead to abnormal fetal development. The placenta is responsible for the exchange of oxygen and nutrients from the mother to the fetus. Normal placentation and placental development are critical for a successful pregnancy. Placental dysfunction is a major cause of pregnancy complications, such as intrauterine growth restriction (IUGR) [1, 2]. Intrauterine growth restriction (IUGR) occurs when the fetus fails to achieve its full growth potential. IUGR is the most important cause of perinatal mortality and morbidity $[3,4]$.

Clinical diagnosis of fetal condition is performed with Doppler Ultrasonography which may provide more in vivo data on disturbances in the 
fetoplacental circulation, but the Doppler measurements do not provide information about the status of blood perfusion within the placenta.

A computer model of the fetoplacental circulation has also proven to be a useful tool in the evaluation of the effects of structural changes [5]. Computational simulations of blood flow are very effective in predicting the performance of circulatory systems in normal as well as in pathophysiological states.

The specific aims of the present study are:

- $\quad$ to develop a technique for hemodynamic computational simulations in placenta vasculature;

- $\quad$ To quantify the blood flow rate in pregnant women and its repartition in placenta based on computational simulations.

\section{Materials and method}

The study used placenta obtained from normal pregnancy of vaginal delivery (age: 31 years old, parity 1, and gestation 38 weeks). The pregnancy is normotensive (median systolic pressure, $110 \mathrm{mmHg}$; median diastolic pressure, $66 \mathrm{mmHg}$ ). Birth weight 2560g. Basal tone in chorionic plate arteries are $2.1 \pm 0.3 \mathrm{kPa}$. After the delivery of the baby and the placenta (Figure 1), the intraplacental (IP) vascular tree was cleared of blood clot. Commercial polymer mixture was injected into the artery to achieve a vascular model demonstrating the intraplacental branching pattern (Figure 2).

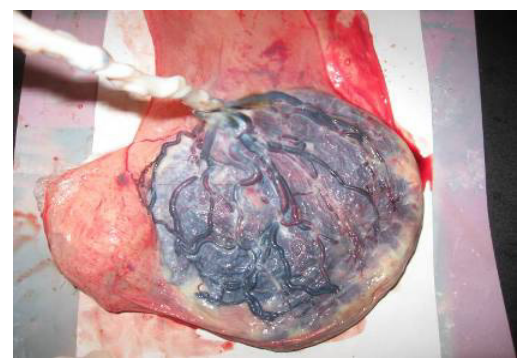

Figure 1: $\quad$ Placenta with the plastic resin in the arterial and venous system before the surrounding tissue has been dissolved by hydrochloric acid.

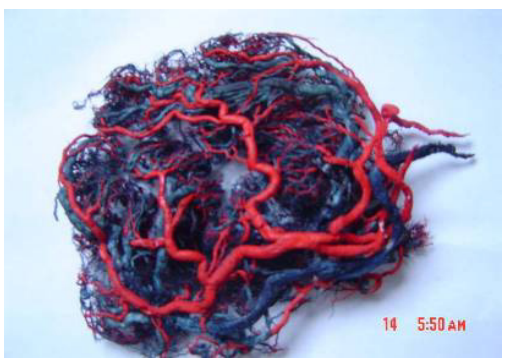

Figure 2: Cast of placenta with a central cord insertion and dominant dichotomous bifurcations.

The polymeric cast of the fetal vasculature of full-term placenta provided information on the geometry of the umbilical, chorionic and intraplacental vessels.

The fetoplacental vasculature is a complex branching network of arteries and veins. The umbilical arteries and vein branch off at the insertion in the placenta into 6-8 generations that traverse over the whole chorionic plate (Figure 2). 
The branching pattern of the chorionic vessels was defined as disperse for a branching network that courses from a central cord insertion, and as magistral for branching vessel that courses from a marginal cord insertion to the opposite edge [6]. The chorionic vessels are relatively large and much smaller vessels branch off this network at almost right angles and penetrate into the placenta toward the maternal side to constitute the IP vessels.

Classification of branching networks in bioengineering and physiology was done by using the definitions of dichotomous and monopodial patterns [7-10] (Figure 3). The dichotomous pattern defines a symmetric network that repeatedly branches into two fairly similar daughter vessels. The monopodial pattern defines a main long mother tube with a fairly constant diameter while small diameter daughter tubes branch off to the sides.
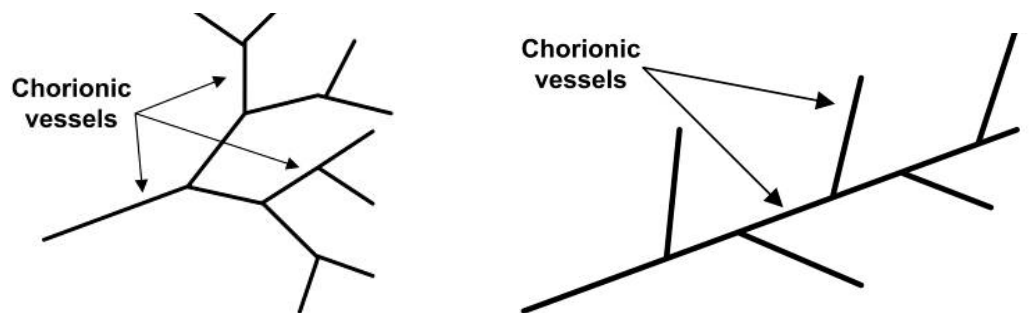

Figure 3: Dichotomous (left) and monopodial (right) branching networks.

Using Doppler ultrasonography, blood flow and systolic/diastolic (S/D) ratios from the umbilical artery were measured at the insertion point of the umbilical cord in placenta (Figures 4 and 5).

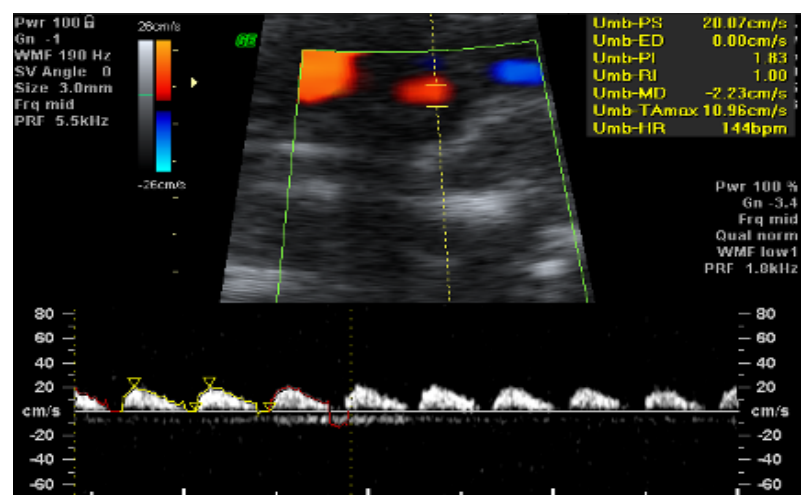

Figure 4: Colour/pulsed Doppler velocity identification at the insertion of the umbilical artery. 


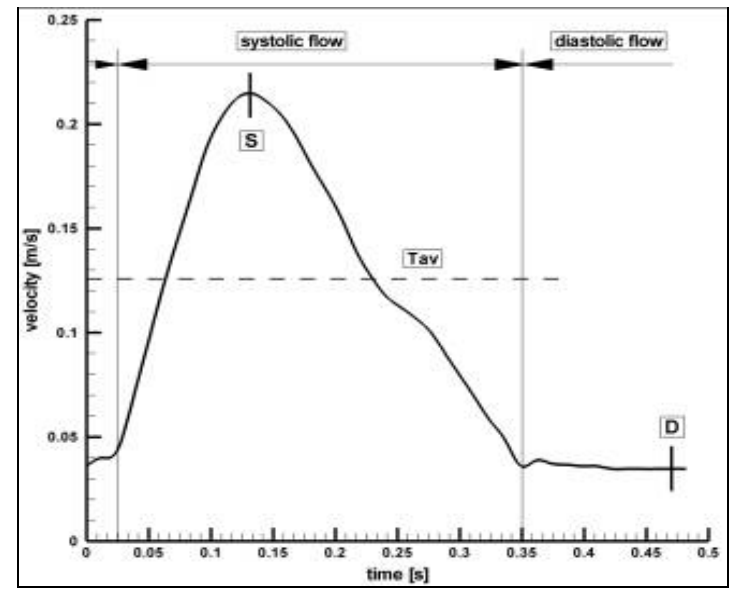

Figure 5: Reconstructed umbilical artery velocity waveform profile at the insertion in placenta. $\mathrm{S}$ - peak systolic velocity, $\mathrm{D}$ - end diastolic velocity, Tav - time average velocity.

\subsection{Computational fluid dynamics}

The blood is assumed to be incompressible, and Newtonian, with dynamic viscosity $(\mu)$ of 0.00408 Pa.s and a density $(\rho)$ of $1050 \mathrm{~kg} / \mathrm{m}^{3}$.

Several recent works investigated the effect of blood rheology assumptions on the axial velocity profiles in bifurcation models and found a satisfactory agreement between the non-Newtonian viscosity and Newtonian viscosity flow based on a characteristic shear rate [11].

The laminar model was used in this study to solve the time dependent 3D Navier-Stokes equations for an incompressible viscous fluid. The blood flow is simulated with the commercial CFD FLUENT 6.3 package [12], parallelized across eight 3.2 GHz Intel processors of a TYANPSC T-650 Rx (Tyan Computer Corporation, Taiwan, parallel computing machine). A second order upwind scheme was used for momentum discretization and the SIMPLEC (Semi-Implicit Method for Pressure-Linked Equations Consistent) was used to achieve pressure-velocity coupling.

The imposed boundary conditions reflect the physiological values obtained by Doppler ultrasonography (Figure 5). Velocity in the umbilical artery before placenta insertion for the investigated patient is $0.32 \mathrm{~m} / \mathrm{s}$, similar to the values presented in [4].

The velocity profiles in the main artery are generally of a parabolic shape (Figure 9). The velocity waveform are shown in Figure 5 are similar with the clinical results in Figure 4. In this case the flow is periodic and mono-directional (i.e. no negative flow) and we used at inlet boundary conditions. The period for the calculations is around $0.5 \mathrm{~s}$ (Figure 5), which corresponds to a heart rate of $120 \mathrm{bpm}$. In a normal fetus the heart rate is typically in the range of $110 \mathrm{bpm}$ to 150 bpm, considerably higher than that in an adult. 
We investigated three different meshes for the computational domain. The number of cells varied between 860,000 and 2,000,000. The mesh was refined in the near-wall region. To model the flow close to the wall, standard wall-function approach is used in the current simulations (Figure 6).
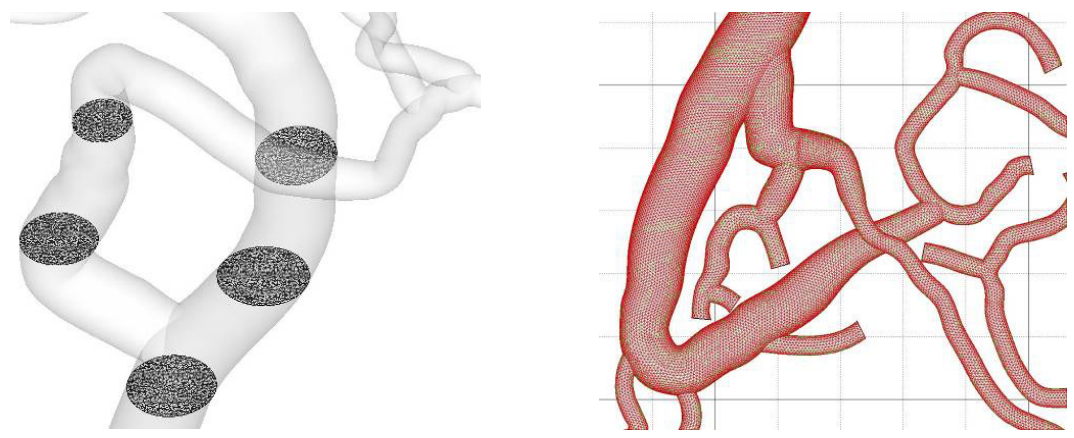

Figure 6: Computational domain discretization.

\section{Results and discussion}

The three-dimensional (3D) geometry of the first placental bifurcation representing a basic dichotomous branching unit (Figure 7) extracted from representative placental cast (Figure 2) are analyzed using computational fluid dynamics techniques.

Commonly, blood velocity profiles are most often described by the pulsatil index (PI) [13], defined as the difference between the maximum and minimum velocities divided by the mean velocity (Figure 5). Abnormal PI in the umbilical artery (UA) is clinically significant because they are associated with adverse pregnancy outcomes $[14,15]$.

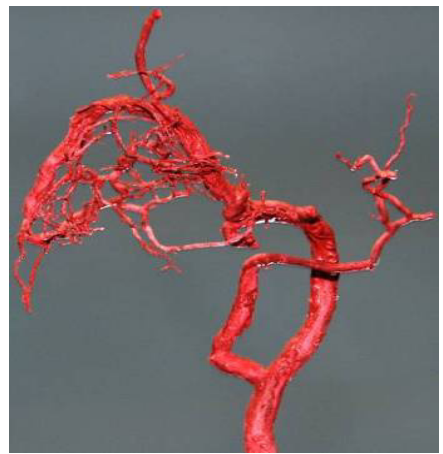

Figure 7: Cast model of the first placental artery bifurcation.

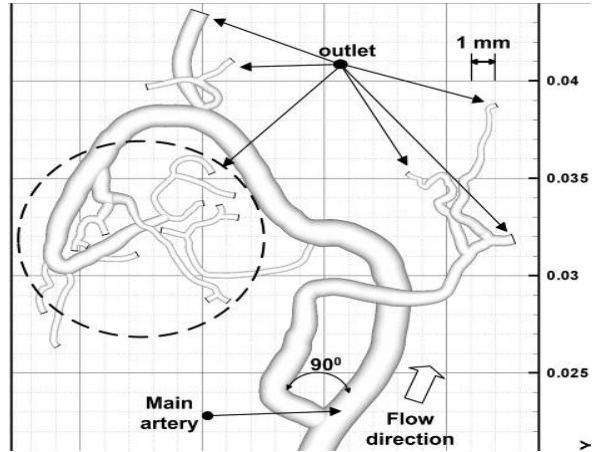

Figure 8:

3D reconstruction of the placental artery cast model and boundary conditions. 
The diameters of the first dichotomous arterial branches ranged from 1.8 to $5.6 \mathrm{~mm}$, and those of the last dichotomous generations near the margins from 0.12 to $1 \mathrm{~mm}$ (Figure 2) with is in good correlation with observation of Gordon [16]. The angle between ramifications and main branches ranging from $60^{\circ}$ to $90^{\circ}$ (Figures 2, 7 and 8). The reconstruction technique adopted in the present study was based on measurements of the 3-D cast model. Moreover, proper boundary conditions were imposed to the computational model in order to avoid influences on the velocity profiles calculated at the region of interest. The computational simulation was conducted for unsteady fetal blood flow in the first bifurcation of the dichotomous branching network of the chorionic arteries (Figure 7).

The primary effort is to provide a comprehensive understanding for the characteristics of the flow fields through the placenta. In this work, the flow fields in the placenta vascularisation are numerically studied in detail for the patient-specific placenta. The dynamics of the flow are revealed by the velocity and pressure fields (Figures 9, 10 and 11).

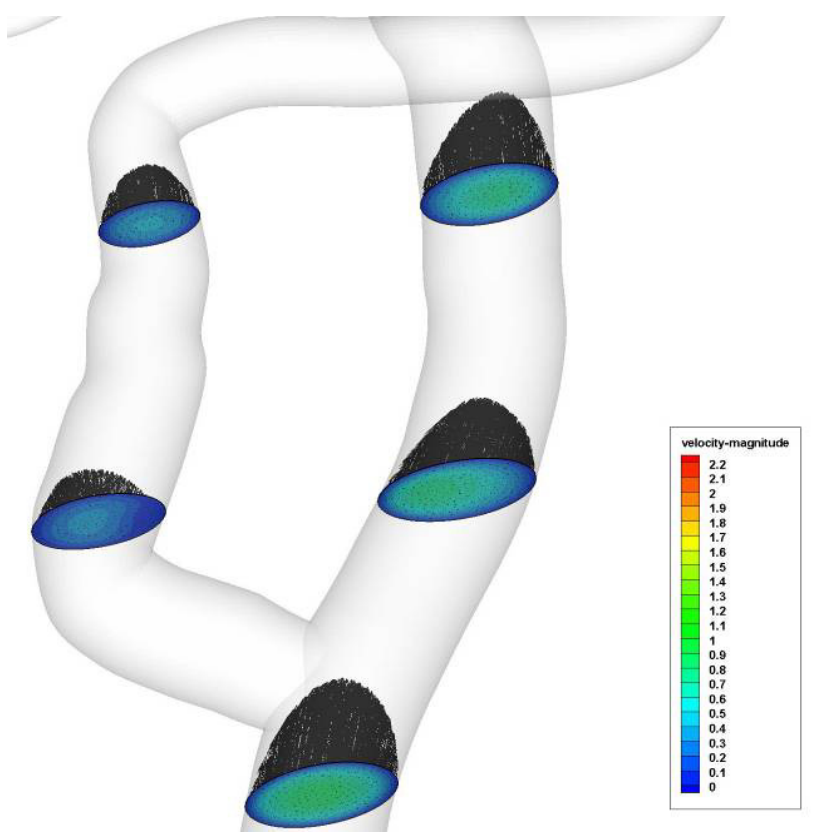

Figure 9: Hemodynamic field in different cross sections of the first bifurcation network at the time $\mathrm{T}=0.12 \mathrm{~s}$ (correspond to the peak systole). Contour plot of the velocity field and de velocity vector field. 


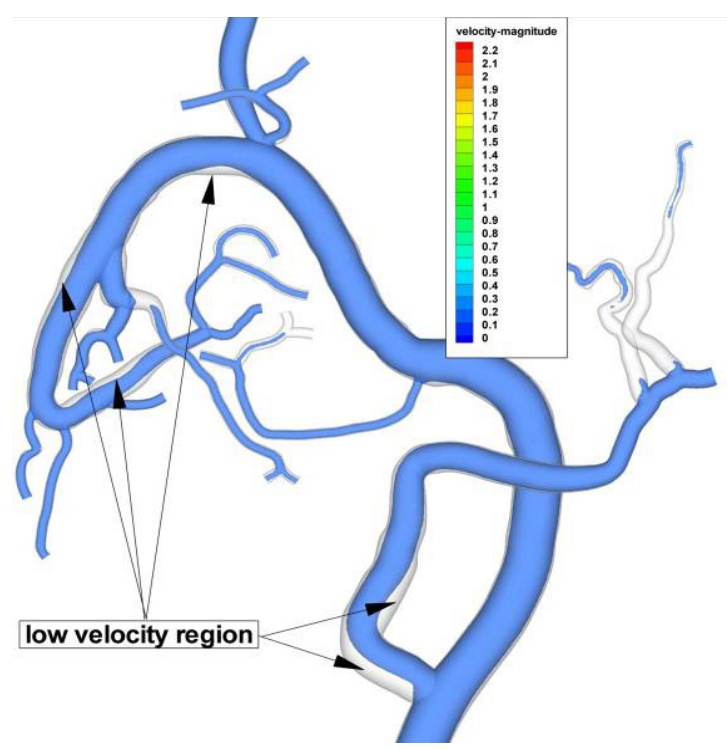

(A)

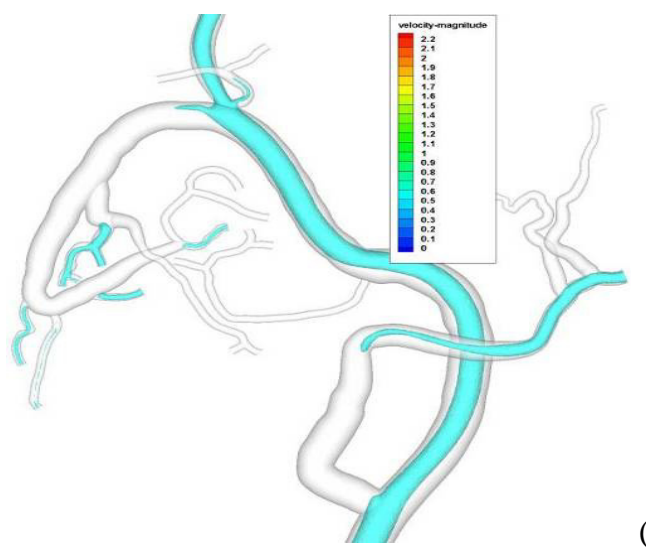

(B)

Figure 10: Iso-velocity contour in the first bifurcation network at the time $\mathrm{T}=0.12 \mathrm{~s}$. (A) iso-velocity contour for $\mathrm{V}=0.15 \mathrm{~m} / \mathrm{s}$; (B) iso-velocity contour for $\mathrm{V}=0.55 \mathrm{~m} / \mathrm{s}$.

The propagation of blood flow and pressure along the fetal arterial tree relates to complex interactions between numerous anatomical, physiological, and rheological parameters. From the point of view of biomedical application, the vessel bifurcation is a place where many diseases start. We are not able to change the geometry of vessel bifurcation, but the understanding of blood flow in it helps us to better cure vessel diseases or to anticipate them.

At a bifurcation the flow in the upstream parent vessel divides into the two daughter vessels so as to bring high velocity blood at the centre of the parent vessel in close proximity to the wall of the flow divider $[7,18]$. When blood 
flows through a diverging bifurcation, a disproportional fraction of red blood cells generally flows into the branch that receives a higher total flow leading to a higher hematocrit in that branch than in the other [19] (Figures 10(A)).

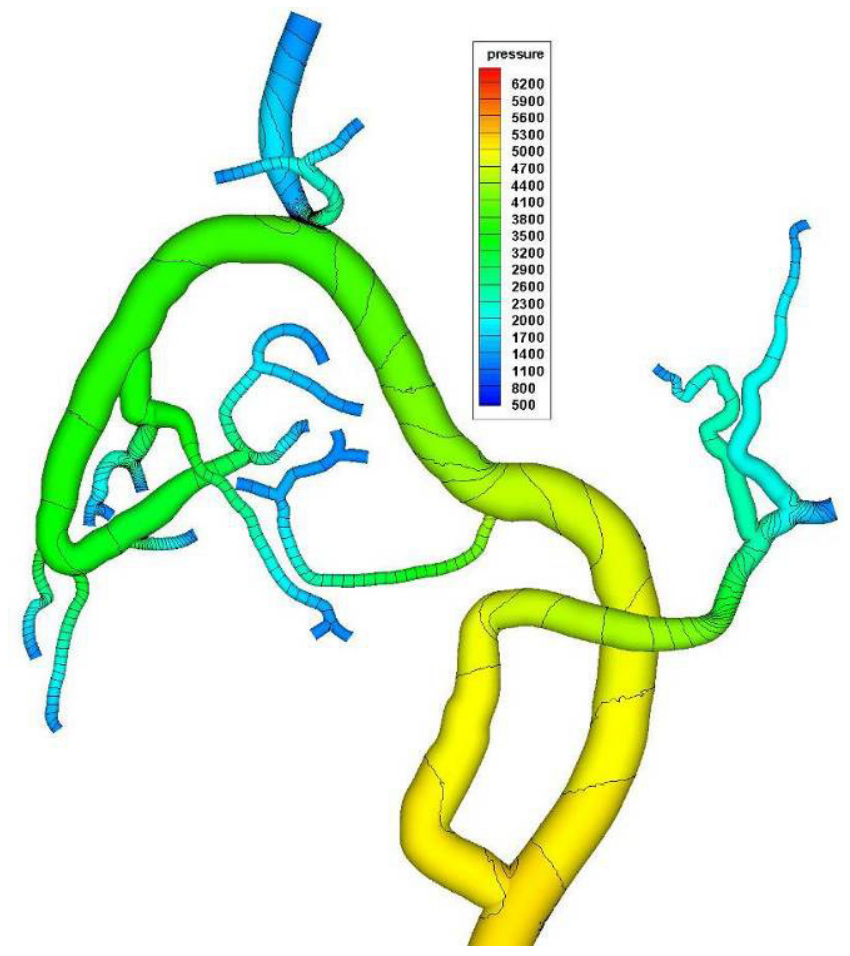

Figure 11: Pressure distribution in the first bifurcation network of the investigated placenta (time $\mathrm{T}=0.12 \mathrm{~s}$ ).

\subsection{Discussion}

The structural organization of the chorionic vasculature was analyzed from cast models of full-term placenta. The model is generated by the corrosion technique used a dental polymer mixture for injection.

Examination of the placental cast provided additional evidence regarding to the architecture of the chorionic vasculature.

This typical geometry is used for model of the vasculature for computational investigations of chorionic blood perfusion. In a bifurcation, the energy dissipation varies from streamline to streamline and the pressure may vary as much over the tube cross section as in the stream wise direction (this observation are in concordance with conclusions of the other group [20]).

Simulation results indicated that generally the velocity profiles in the placental vessels, both immediately upstream and downstream the bifurcation, are close to parabolic profiles (Figure 9). The small differences appear in this 
region due to the not completely developed profile and vessel curvature. These results are not surprising according to the high length and relatively straight path of the main artery. The specific literature which investigated the velocity profiles throughout the branches of a bifurcation clearly showed skewed profiles with peculiar shapes depending on the branching geometry (Figure 10), the flow conditions and the distance from the branching point [21].

The pressure distribution along the longitudinal direction of the first bifurcation network is depicted in Figure 11. Pressure distribution is uniform, and have an average value about $\mathrm{P}=5,300 \mathrm{~Pa}$. In cross sections upstream and downstream of the each bifurcation region the pressure differences are approximately $300 \mathrm{~Pa}$.

\subsection{Study limitations}

Some limitations of our study should be pointed out. First, the placenta movement and the movement of the placental arteries cannot be simulated yet, therefore, not included in the present numerical analysis. Second, we modelled only the first bifurcation of the dichotomous branching network of the chorionic arteries. Third, in this model vessel walls are assumed rigid.

\section{Conclusions}

During pregnancy there are large-scale changes of physiological function affecting all body systems. Alterations in pregnancy hemodynamic start early in pregnancy and are maintained to the third trimester.

Computational simulations of blood flow have been shown to be very effective in predicting the performance of circulatory systems in normal as well as in pathophysiological states.

The computational results together with the echo-Doppler measurements allowed us to quantify the uterine blood flow rate in pregnant women and its repartition in placenta branches.

The clinical application of simulation based medical planning techniques is a fundamentally new approach to treatment planning. In the case of placenta disease, these methods could enable physicians to develop the patient-specific treatment plans in order to improve blood flow.

\section{References}

[1] L. Poston, The control of blood to the placenta, Experimental Physiology, 82, 377-387, 1997.

[2] T. Kiserud, O. Kilavuz, L.R. Hellevik, Venous pulsation in the left portal branch: the effect of pulse and flow direction, Ultrasound Obstet Gynecol, 21, 359-364, 2003.

[3] G. Larciprete, H. Valensise, G. Di Pierro, B. Vasapollo, B. Casalino, D. Arduini, et al. Intrauterine growth restriction and fetal body composition, Ultrasound Obstet Gynecol, 26, 258-262, 2005. 
[4] K. Marsal, Intrauterine growth restriction, Curr Opin Obstet Gynecol, 14, 127-135, 2002.

[5] A. Jafari, S.M. Mousavi, P. Kolari, Numerical investigation of blood flow. Part I: In micro vessel bifurcations, Communications in Nonlinear Science and Numerical Simulations, 13, 1615-1626, 2008.

[6] K. Benirschke, P. Kaufmann, Pathology of the Human Placenta, SpringerVerlag. New York, 1995.

[7] S. Aharinejad, W. Schreiner, F. Neumann, Morphometry of human coronary arterial trees, Anat. Rec., 251, 50-59, 1998.

[8] J.C. Parker, C.B. Cave, J.L. Ardell, et al. Vascular tree structure affects lung blood flow heterogeneity simulated in three dimensions, J. Appl. Physiol. 83, 1370-1382, 1997.

[9] M. Zamir, Distributing and delivering vessels of the human heart, J. Gen. Physiol., 91, 725-735, 1998.

[10] M. Zamir, Fractal dimensions and multifractility in vascular branching, J. Theor. Biol., 212, 183-190, 2001.

[11] S. Shibeshi, W.E. Collins, The rheology of blood flow in a branched arterial system, Appl. Rheol., 15(6), 398-405, 2005.

[12] Fluent 6.3, Ansys Fluent Incorporated, 2006.

[13] R.G. Gosling, D.H. King, Ultrasound angiology. In: Arteries and Veins, Marcus AW and Adamson L. Edinburgh, UK: Churchill Livingstone, 1975.

[14] V.H.M. Karsdorp, J.M.G. Van Vugt H.P. Van Geijn, P.J. Kostense, D. Arduini, N. Montenegro, T. Todros, Clinical significance of absent or reversed end diastolic velocity waveforms in umbilical artery. Lancet, 344, 1664-1668, 1994.

[15] J. W. Wladimiroff, J.A. Van den Wijngaard, S. Degani, M.J. Noordam, J. Van Eyck, H.M. Tonge. Cerebral and umbilical arterial blood flow velocity waveforms in normal and growth-retarded pregnancies. Obstet Gyneco, I 69, 705-709, 1987.

[16] Z. Gordon, O. Eytan, A.J. Jaffa, D. Eland, Fetal blood flow in branching models of the chorionic arterial vasculature, Ann. N.Y. Acad. Sci., 1101, 250-265, 2007

[17] L.R. Hellevik, T. Kiserud, F. Irgens, T. Ytrehus, S.H. EIK-NES, Simulation of pressure drop and energy dissipation for blood flow in a human fetal bifurcation, Journal of Biomedical Engineering, 120, 455-462, 1998.

[18] C.H. Brucker, M.L. Riethmuller, Cyclic oscillations in a system of repeatedly branching channels, Physics of Fluids, 10 (4), 877-885, 1998

[19] J.A. Arroyo, V.D. Winn, Vasculogenesis and angiogenesis in the IUGR placenta, Seminars in Perinatology, 32, 172-177, 2008.

[20] T. Pedley, The Fluid Mechanics of Large Blood Vessels, Cambridge University Press, 1980.

[21] J. Chen, X.Y. Lu, Numerical investigation of the non-Newtonian blood flow in a bifurcation model with a non-planar branch, J. Biomech., 37(12), 1899-1911, 2004. 